\title{
Genome and transcriptome analysis of the beet armyworm Spodoptera exigua reveals targets for pest control
}

\author{
Sabrina Simon, ${ }^{1, t, *}$ Thijmen Breeschoten, ${ }^{1, \dagger}$ Hans J. Jansen, ${ }^{2}$ Ron P. Dirks, ${ }^{2}$ M. Eric Schranz, ${ }^{1}$ and Vera I. D. Ros ${ }^{3, t, *}$ \\ ${ }^{1}$ Biosystematics Group, Wageningen University \& Research, 6708 PB Wageningen, The Netherlands, \\ ${ }^{2}$ Future Genomics Technologies, Leiden, The Netherlands and \\ ${ }^{3}$ Laboratory of Virology, Wageningen University \& Research, 6708 PB Wageningen, The Netherlands \\ *Corresponding authors: Biosystematics Group, Wageningen University \& Research, Droevendaalsesteeg 1, 6708 PB Wageningen, The Netherlands. Email: \\ sabrina.simon@wur.nl (S.S.) and Laboratory of Virology, Wageningen University \& Research, Droevendaalsesteeg 1, 6708 PB Wageningen, The Netherlands. Email: \\ vera.ros@wur.nl (V.I.D.R.) \\ ${ }^{\dagger}$ These authors contributed equally to this work.
}

\begin{abstract}
The genus Spodoptera (Lepidoptera: Noctuidae) includes some of the most infamous insect pests of cultivated plants including Spodoptera frugiperda, Spodoptera litura, and Spodoptera exigua. To effectively develop targeted pest control strategies for diverse Spodoptera species, genomic resources are highly desired. To this aim, we provide the genome assembly and developmental transcriptome comprising all major life stages of $S$. exigua, the beet armyworm. Spodoptera exigua is a polyphagous herbivore that can feed on $>$ 130 host plants, including several economically important crops. The $419 \mathrm{Mb}$ beet armyworm genome was sequenced from a female $S$. exigua pupa. Using a hybrid genome sequencing approach (Nanopore long-read data and Illumina short read), a high-quality genome assembly was achieved (N50=1.1 Mb). An official gene set (18,477 transcripts) was generated by automatic annotation and by using transcriptomic RNA-seq datasets of $18 \mathrm{~S}$. exigua samples as supporting evidence. In-depth analyses of developmental stage-specific expression combined with gene tree analyses of identified homologous genes across Lepidoptera genomes revealed four potential genes of interest (three of them Spodoptera-specific) upregulated during first- and third-instar larval stages for targeted pest-outbreak management. The beet armyworm genome sequence and developmental transcriptome covering all major developmental stages provide critical insights into the biology of this devastating polyphagous insect pest species worldwide. In addition, comparative genomic analyses across Lepidoptera significantly advance our knowledge to further control other invasive Spodoptera species and reveals potential lineagespecific target genes for pest control strategies.
\end{abstract}

Keywords: Beet armyworm; Spodoptera exigua; whole genome; transcriptomics; gene expression; pest control

\section{Introduction}

Analysis of genome and transcriptome data can be used to study many important questions ranging from species-specific mutations to comparative genomic evolutionary patterns. The genus Spodoptera is known for the high number of notorious pest species causing enormous agricultural damage resulting in economic losses worldwide, including Spodoptera exigua, Spodoptera frugiperda, and Spodoptera litura (Pogue 2002; Goergen et al. 2016; Cheng et al. 2017; EPPO 2017). The beet armyworm, S. exigua (Hübner) (Lepidoptera: Noctuidae) is a devastating polyphagous insect pest with a worldwide distribution (Mehrkhou et al. 2012; Fu et al. 2017), being able to feed on more than 130 plant species from at least 30 families including several economically important crops such as sugar beet, cotton, soybean, cabbage, maize, and tomato (Merkx-Jacques et al. 2008; Robinson et al. 2010a; Mehrkhou et al. 2012; Fu et al. 2017). Spodoptera exigua originated in Southern Asia and was subsequently introduced to other parts of the world including North America and Europe (Mehrkhou et al. 2012; Fu et al.
2017). It is widely distributed in the tropical and subtropical regions and migrates into more temperate regions throughout the growing season (Pogue 2002). Its long-distance migration likely played a major role in the geographic expansion of populations and its spread across the world (Fu et al. 2017). In temperate regions, it can be abundant in greenhouses (Smits et al. 1986).

Successful control of S. exigua is challenging due to its broad host range, rapid growth rate, its migratory dispersal and its ability to rapidly evolve resistance to pesticides (Fu et al. 2017; Hu et al. 2021; Huang et al. 2021). Moreover, the use of conventional chemical pesticides causes health and environmental issues and is generally less accepted (Wheeler 2002; Omkar 2016). Therefore, there is a pressing need for other, more sustainable, strategies to control S. exigua and other Spodoptera species. A promising approach includes RNA interference (RNAi)-based insect management (Burand and Hunter 2013; Scott et al. 2013; Renuka et al. 2017). One of the major challenges is to find target genes for RNAi to control specific pest species or a range of closely related pest species (Li et al. 2013; Bi et al. 2016; Tian et al. 2019). One way to 
select potential lineage-specific candidate genes is by carefully analyzing homologous relationships of genes in related species. Targeting specific gene(s) of single species using RNAi approaches could be an extremely powerful tool to diminish a specific pest outbreak without harming other (closely related) arthropod species (Price and Gatehouse 2008; Scott et al. 2013), which often does occur when applying general insecticides (Schulz 2004). Given the high pest potential of many Spodoptera species, lineagespecific genes should be identified that can be targeted during pest outbreaks. However, genomic studies have been focused mainly on S. frugiperda (Kakumani et al. 2014; Gouin et al. 2017), whereas other Spodoptera species have largely been neglected. To address this gap, we present the $S$. exigua genome assembly and official gene set (OGS).

In this study, we obtained an RNA-sequencing (RNA-seq) profile across all major life stages of S. exigua. We performed an indepth analysis of gene expression patterns during the different developmental stages. We identified four candidate genes for RNAi-based pest management strategies, and additionally confirmed Spodoptera-specificity for three of them. Furthermore, we produced a de novo assembled genome draft of S. exigua, based on one female pupa.

\section{Materials and methods Breeding and sample collection}

Spodoptera exigua specimens originated from a stock rearing of the Laboratory of Virology, Wageningen University \& Research, which was initiated in July 2014 using pupae from a large continuous rearing, kindly provided by Andermatt Biocontrol (Switzerland). The rearing was kept on an artificial diet at $27^{\circ} \mathrm{C}$ with $50 \%$ relative humidity and a $14: 10 \mathrm{~h}$ light:dark photoperiod. The artificial diet consisted of water, cornflour, agar, yeast, wheat germ, sorbic acid, methylparaben, ascorbic acid, and streptomycin sulfate. Disposable plastic trays covered with paper tissues and a lid were used as rearing containers for groups of maximum 35 larvae (for larger stages). Late fifth instars were transferred to a plastic tray containing vermiculite to facilitate pupation. Pupae were collected and transferred to cylindrical containers lined with paper sheets for egg deposition, with around 45 pupae per cylinder. Adult moths were provided with water. Collected eggs were surface sterilized with formaldehyde vapor to eliminate external microbial contamination.

High-molecular weight (HMW) chromosomal DNA was extracted from a female S. exigua pupa using the Qiagen Genomic-tip 100/G kit according to the manufacturer's instructions (Qiagen, Venlo, The Netherlands). The quality of the extracted HMW DNA was analyzed on an Agilent 4200 TapeStation System using Genomic DNA ScreenTape (Agilent, Amstelveen, The Netherlands).

To retrieve samples for RNA-Seq, a newly hatched male and female from the continuous rearing were mated in a plastic cup. Offspring of this couple was used for RNA-Seq, six stages were collected: embryos (eggs), first-instar larvae, third-instar larvae, pupae, male adults, female adults, with three replicates (individuals) per stage except for the embryonic stage were three clusters of each 100 eggs were taken. To obtain the samples, eggs were harvested, and larvae were reared as above. For the embryonic stage, egg clusters (laid within $21 \mathrm{~h}$ ) were cut out of paper, transferred to Eppendorf tubes, snap frozen in liquid nitrogen and transferred to $-80^{\circ} \mathrm{C}$ until shipment on dry ice to Future Genomics Technologies for further RNA extraction and sequencing. Synchronized newly hatched (non-fed) first-instar larvae, early third-instar larvae, second day pupae, and newly emerged (non-mated) female and male adults were collected. Individuals were transferred to Eppendorf tubes and snap frozen as before. For an overview of all samples please refer to Supplementary Table S1. Please also refer to Figure 1 for an overview of the developmental stages.

\section{Sequencing and assembly of the Spodoptera exigua genome}

A dual sequencing approach was used for de novo assembly of the S. exigua genome sequence. In total, $\sim 100 \mathrm{~Gb}$ of raw Nanopore long-read data (Oxford Nanopore Technologies, Oxford, UK) and $\sim 73 \mathrm{~Gb}$ of raw Illumina $2 \times 150$ nt short-read data were generated. Long sequence read data were generated using the Oxford Nanopore Technologies platform. Prior to library preparation, HMW DNA was sheared to $\sim 12.5 \mathrm{~kb}$ fragments using Covaris gTUBE (Covaris Inc., Woburn, MA, USA). Quality was checked on the Agilent TapeStation. Library preparation was done with the SQK-LSK109 1D ligation kit from Oxford Nanopore Technologies (ONT). Samples were sequenced using one run on an ONT MinION R9.4.1 flowcell and one run on an ONT PromethION R9.4.1 flowcell, respectively. Basecalling was done with Guppy v2.2.2 (ONT MinION) and v1.6.0 (ONT PromethION), respectively. Basecalled reads were used for further processing and assembly.

In addition to long sequence read data, short-read data were generated using the Illumina NovaSeq 6000 system. Library preparation was done with the Nextera DNA Flex Library Prep Kit following manufacturers' protocol (Illumina Inc. San Diego, CA, USA) and quality was checked using the Agilent Bioanalyzer 2100 High Sensitivity DNA Kit (Agilent, Amstelveen, The Netherlands). The genomic paired-end (PE) library was sequenced with a read length of $2 \times 150 \mathrm{nt}$. Image analysis and basecalling were done by the Illumina pipeline. Please refer to Supplementary Table S2 for an overview of the DNA sequencing approach. All raw reads from the Illumina, MinION, and PromethION sequencing runs were submitted to the NCBI SRA database under accession number PRJNA623582 under sample number SAMN14550570.

To assemble the S. exigua genome sequence, only long sequence read data were used. First, all reads with a quality score lower than qv $=7$ were removed from the long sequence read dataset. Then, the SEA program (Future Genomics Technologies BV, Leiden, The Netherlands; Jansen et al. 2017; program provided at the Dryad digital repository) was used to prepare seed sequences from the longest reads. In total, $\sim 30 \times$ estimated coverage of the longest reads was then aligned to these seeds. Reads, alignments, and seed files were used to run Tulip v. 1.0.0 (Future Genomics Technologies BV, Leiden, The Netherlands; Jansen et al. 2017; program provided at the Dryad digital repository) to obtain an assembly. The assembly results were used to further optimize the assembly parameters. After this optimization, the total size of the assembled genome was $419 \mathrm{Mb}$, which was divided over 946 contigs (largest contig $=4.08 \mathrm{Mb}$ ) with a contig $\mathrm{N} 50$ of $1.10 \mathrm{Mb}$. To further optimize the genome assembly, Racon (Loman et al. 2015) was used (two rounds) to correct mistakes in the assembly and then two rounds of Pilon polishing (Walker et al. 2014; Goodwin et al. 2015) were used to polish the assembly based on the genomic Illumina reads and to reach a high accuracy of the de novo assembly that was the basis for genome annotation. The final genome assembly was submitted to the NCBI GenBank database and is available under accession JACEFF000000000, version JACEFF010000000 is used in this study. As a quality check, the Benchmarking Universal Single-Copy Ortholog (BUSCO v. 3.0.2; Seppey et al. 2019) analysis was done on the polished de novo assembly using the "insecta_odb9" dataset. 


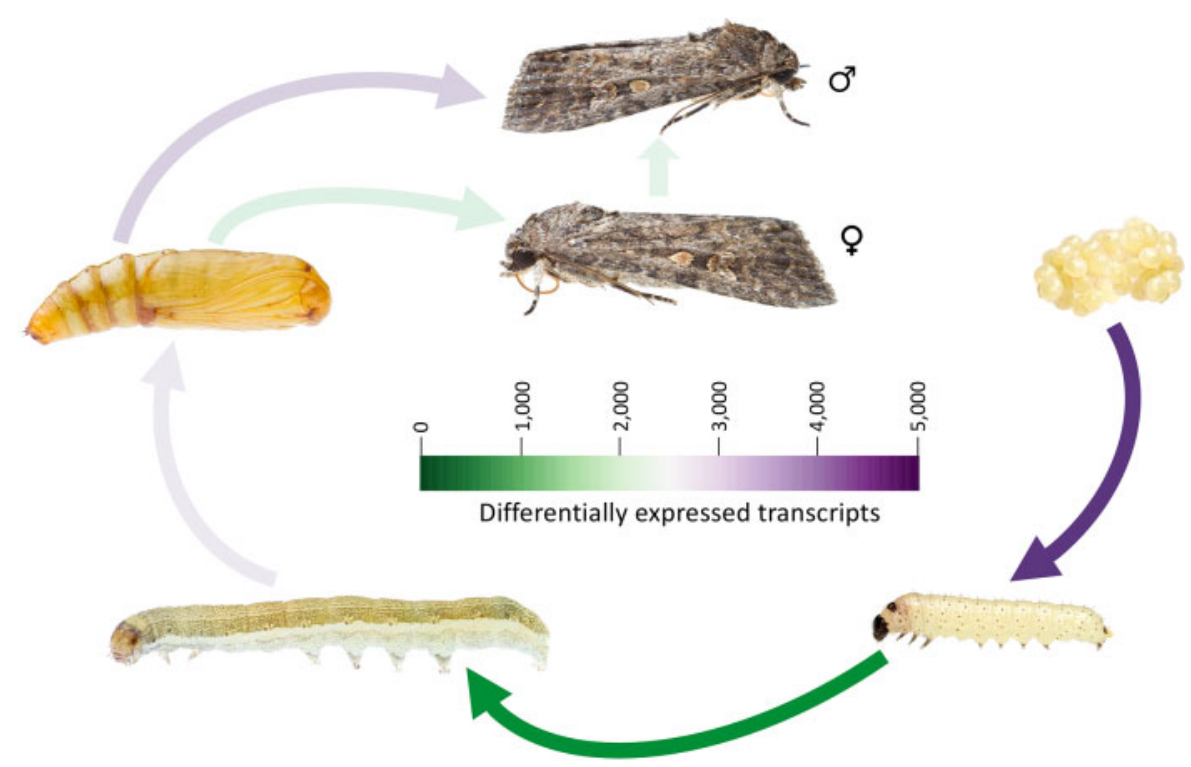

Figure 1 Spodoptera exigua life cycle and gene expression profile. The major developmental stages and sexes sequenced for S. exigua are shown, starting from an egg (embryonic stage) and proceeding two larval stages, namely first and third instar. After the pupal stage, there is the final differentiation into adult male and female. The color of the arrows is proportional to the number of statistically significant DE genes (FDR $=0.001$, minimal foldchange of four). Note that the size of the developmental stages is not proportional.

\section{Sequencing the developmental transcriptome of Spodoptera exigua}

Following the Illumina Truseq-stranded mRNA library prep protocol (150-750 bp inserts), we prepared 18 different indexed RNA-Seq libraries representing the different developmental stages, namely embryonic stage, early first-instar larva, early third-instar larva, pupa, adult (female and male), and including three biological replicates per stage/sex (Supplementary Table S1.1). Libraries were sequenced on an Illumina NovaSeq 6000 system at an average of 13.4 million PE2x150nt reads (6.9-22.5 million reads) per sample at Future Genomics Technologies BV, Leiden, The Netherlands. For an overview of the number of raw reads per sample please refer to Supplementary Table S1.3. The sequencing reads were quality checked using FastQC v. 0.10.1 (Andrews 2010). Adapter sequences were removed and quality-filtered using Trimmomatic v. 0.36 (Bolger et al. 2014), with parameters set: TruSeq3-PE-2.fa : 2:30:10, LEADING: 5, TRAILING: 5, SLIDINGWINDOW : 4:20, and removing all reads of $<36 \mathrm{bp}$ in length. All raw reads from the Illumina RNASeq approach were submitted to the NCBI SRA database under accession number PRJNA623582.

\section{Annotation of the Spodoptera exigua genome sequence}

The assembled and polished genome was annotated using the maker3 pipeline (maker-3.01.02-beta). As the first step in this analysis, a repeat library was constructed with RepeatModeler (RepeatModeler-open-1.0.11; -database Spodoptera_exigua). This species-specific library was used in addition to the RepeatMasker library (Lepidoptera). For gene prediction, Augustus v. 3.3.2 was used which used the model from heliconius_melpomene1 to find genes. As additional evidence for gene models, the protein sequences for the family of the Noctuidae were extracted from UniProt (accessed March 7, 2019). Also, the RNA-Seq datasets of our 18 S. exigua samples were used as supporting evidence. This dataset was first assembled using the De Bruijn graph-based de novo assembler implemented in the CLC Genomics Workbench version 4.4.1 (CLC bio, Aarhus, Denmark). The available S. exigua
mRNA nucleotide data from NCBI Genbank (accessed March 7, 2019) was added to this data. After running the pipeline, maker3 annotated a total of 18,477 transcripts. Gene annotations, predicted messenger RNA (mRNA) and proteins, and assemblies for gene annotations are also provided at the Dryad digital repository.

Spodoptera exigua proteins from the OGS v. 1.1 were further annotated using InterProScan (v. 5.36-75) with several approaches including Gene Ontology (GO) term annotation (Jones et al. 2014). Of the 18,477 transcripts, 16,718 transcripts retrieved annotations (Supplementary Table S3). Furthermore, the transcript OGS was used in a local BLASTX search v. 2.6.0 (Camacho et al. 2009; max_hsps 1, best_hit_overhang 0.1 and E-value cutoff $\leq 1 \mathrm{e}-3$ ) against a locally constructed database of all Arthropoda protein sequences downloaded from the NCBI protein database (accessed, January 31, 2019). The translated proteins were additionally used in a BLASTP search v. 2.6.0 (Camacho et al. 2009) against the same Arthropoda database and parameters (Supplementary Tables S4 and S5).

\section{Transcript expression quantification}

To estimate transcript expression, reads of all samples from each developmental stage were separately mapped to the newly generated S. exigua genome (version JACEFF010000000) using Bowtie2 v. 2.3.4 (Langmead and Salzberg 2012). The isoform and gene abundance estimations were done using RSEM v. 1.3.0 (Li and Dewey 2011). A raw (nonnormalized) count matrix was created using the perl script "abundance_estimates_to_matrix.pl" implemented in the Trinity v. 2.5.1 package (Grabherr et al. 2011). The count matrix was cross-sample normalized using the "calcNormFactors" function in edgeR v.3.20.8 (Robinson et al. 2010b; R v. 3.4.3) using trimmed mean of $M$ values (TMM; Robinson and Oshlack 2010). See Supplementary Table S6 for the raw counts matrix of isoforms in the samples. The normalized count matrix was further filtered by abundance based on countper-million values (CPM; to account for library size differences between samples) using edgeR v. 3.20.8 (Robinson et al. 2010b). Only genes with a minimum of five counts in at least two of the 
samples were considered expressed and retained in the dataset (see Supplementary Table S7).

To measure the similarity of the samples covering the developmental stages and to verify the biological replicates, we implemented the trinity-provided perl script "PtR." The PCA plot is generated based on the raw nonnormalized isoform count matrix which we centered, CPM normalized, log transformed and filtered using a minimum count of 10 (Supplementary Figure S1).

The differential expression analysis was performed using DESeq2 v. 1.18.1 (Love et al. 2014) as implemented in the Trinity package. Transcripts were considered differentially expressed (DE) with a minimal fold-change of four between any of the treatments and a false discovery rate (FDR) of $P$-value $\leq 1 \mathrm{e}-3$. The CPM and TMM normalized expression values of all DE transcripts were hierarchically clustered and cut at 50\% using the Trinityprovided script "define_clusters_by_cutting_tree.pl." This resulted in 14 clusters of DE transcripts with similar expression patterns that were used in the cluster-specific GO analysis.

See Supplementary Table S8 for an overview of cluster membership of all 9896 DE isoforms and Figure 2 and Supplementary Figure S2 for expression patterns.
GO analysis was performed using the GOseq package using the Trinity-provided script "runGOseq.R," adjusting for transcript length bias in deep sequencing data (Young et al. 2010) and using the GO annotation retrieved from the Interpro annotation. See Supplementary Table S9 for an overview of GO annotations within the clusters. For the identified DE genes, statistically overrepresented GO terms in each cluster were identified using FDRadjusted $P$-value $(<0.05)$ and were further summarized to generic GO slim categories (Figure 3 and Supplementary Table S10) using the R package GOstats (Falcon and Gentleman 2007). R script for summarizing GO slim categories is provided at the Dryad digital repository.

\section{Phylogenomic analyses and comparative genome analyses}

We used BUSCO v. 4.0.5 applying the insecta_odb10 as a reference lineage dataset (Seppey et al. 2019) and comprising in total 1367 BUSCOSs, to extract single copy complete BUSCOs on the amino acid (aa) level for S. exigua and another 36 lepidopteran genomes (Supplementary Table S11).

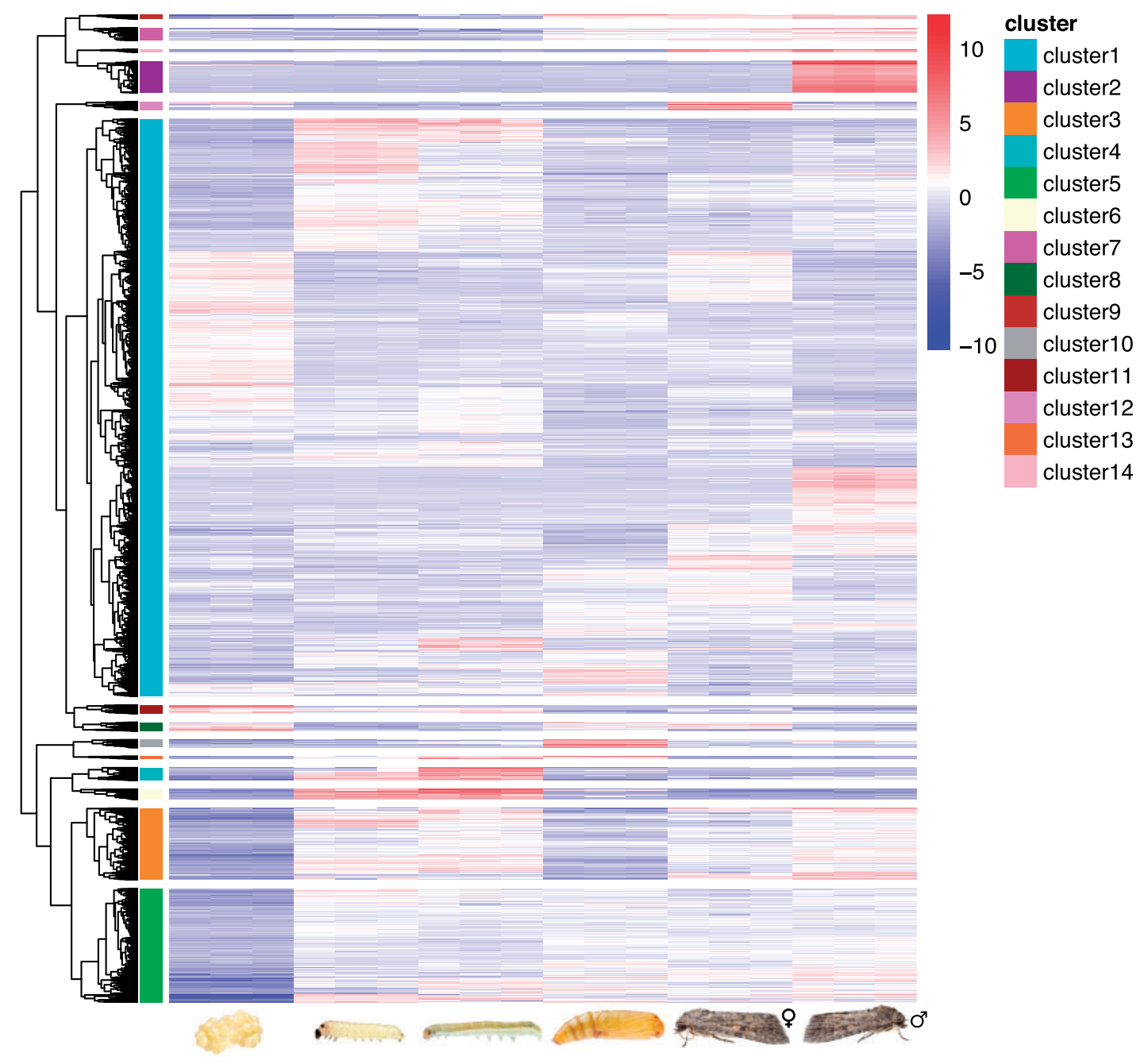

Figure 2 Hierarchical clustering dendrogram of all DE genes in the life cycle of Spodoptera exigua. Heatmap shows 9896 transcripts which have been identified DE (minimal fold-change of four, FDR $\leq 1 \mathrm{e}-3$ ) between the six developmental stages/sexes including three replicates each (left to right: embryo, first-, third-instar larva, pupa, female adult, male adult). Transcripts from 14 distinct clusters using a cutoff at 50\% (right dendrogram). The color key of the heatmap indicates low (blue) to high (red) expression values for transcripts. 


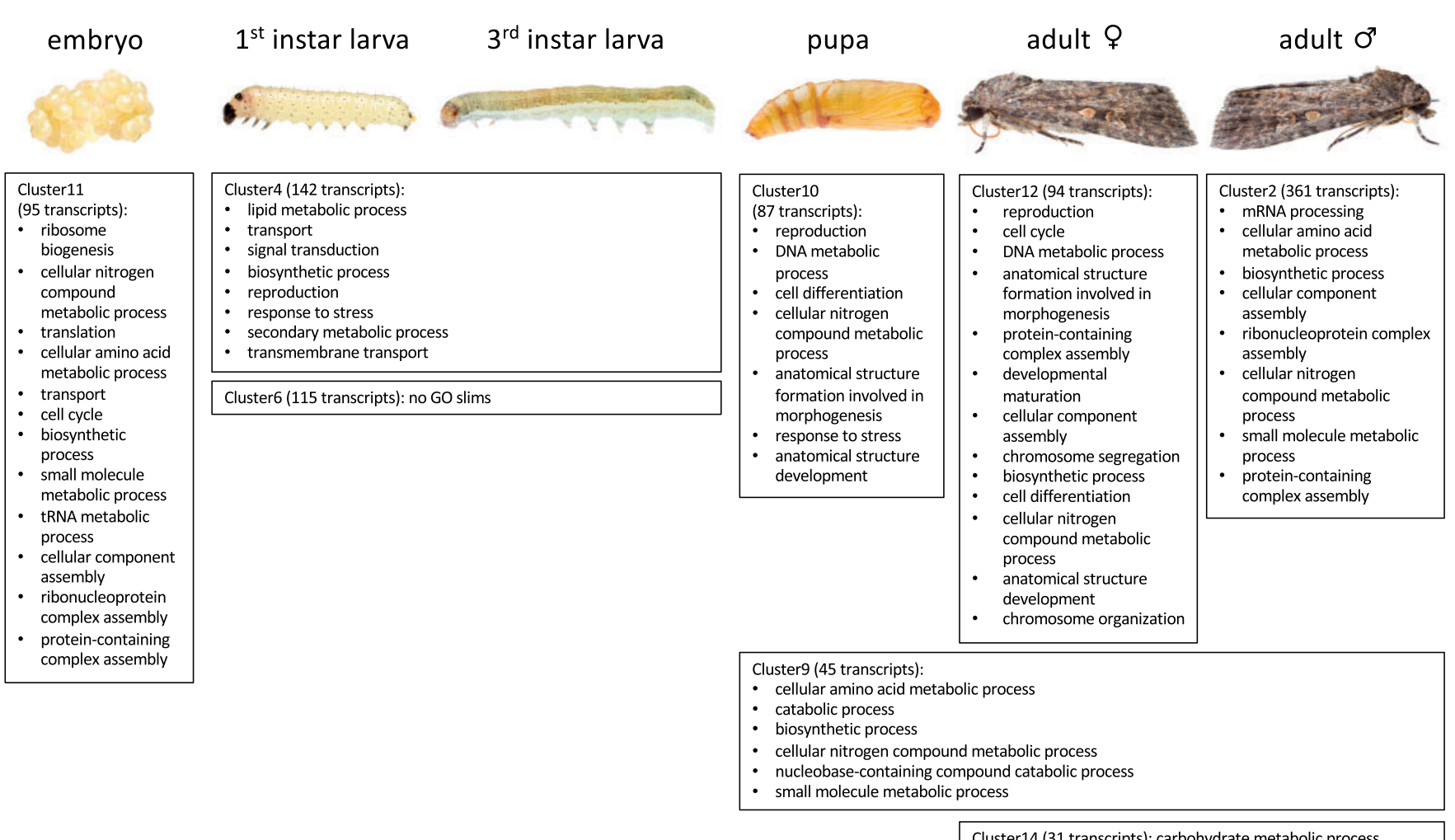

Cluster14 (31 transcripts): carbohydrate metabolic process

Figure 3 Upregulated GO slims (Biological Process) per development stages. Shown are only the eight clusters of DE transcripts that could be assigned to one developmental stage or sex or to subsequent developmental stages. The cluster number is according to the formed clusters as indicated in Figure 2. The number of transcripts is provided in parentheses as well as the statistically overrepresented GO terms (FDR $\leq 0.05)$ which have been summarized to generic GO slim categories.

For the phylogenomic analysis, first, aa sequences of singlecopy BUSCO genes were separately aligned using MAFFT v. 7.305 (Katoh and Standley 2013) using the L-INS-i algorithm. For the identification of putative ambiguously aligned or randomized multiple sequence alignment (MSA) sections, we used Aliscore v. 1.2 (Misof and Misof 2009; Kück et al. 2010) on each MSA with the default sliding window size, the maximal number of pairwise sequence comparisons and a special scoring for gap-rich aa data (options - $r$ and -e). After exclusion of the identified putative ambiguously aligned or randomized MSA sections with ALICUT v. 2.3 (Kück et al. 2010), the final MSAs were concatenated into supermatrices using FASconCAT-G v. 1.02 (Kück and Longo 2014). The resulting dataset comprised 1367 gene partitions and 687,494 aa positions.

Prior to the tree reconstruction, the best scoring aa substitution matrix for each gene partition was selected with ModelFinder as implemented in IQ-TREE v. 1.6 .12 (Kalyaanamoorthy et al. 2017). We restricted the search of the best fitting model to eight aa substitution matrices appropriate for nuclear markers: DCMut (Kosiol and Goldman 2005), JTT (Jones et al. 1992), LG (Le and Gascuel 2008), Poisson, PMB (Veerassamy et al. 2003), VT (Muller and Vingron 2000), and WAG (Whelan and Goldman 2001). We additionally included the protein mixture model LG4X (Le et al. 2012), which accounts for FreeRate heterogeneity. Furthermore, we allowed testing the default rate heterogeneity types (E, I, G, I+ G, and FreeRates: R; Gu et al. 1995; Soubrier et al. 2012; Yang 1994), with or without empirical rates (-F, -FU) as well as testing the number of rate categories (-cmin 4 - cmax 15). The best model for each gene partition was selected according to the best second-order or corrected Akaike Information Criterion score (Hurvich and Tsai 1989). Dataset and partition scheme including selected models are provided at the Dryad digital repository.

Phylogenetic relationships were inferred under the maximum likelihood (ML) optimality criterion as implemented in IQ-TREE v. 1.6.12 (Chernomor et al. 2016; Nguyen et al. 2015) using the best scoring aa substitution matrix for each gene partition and the edge-proportional partition model allowing partitions to have different evolutionary rates (option -ssp). We performed 50 independent tree searches (25 searches with a random and 25 with a parsimony start tree). The resulting number of unique tree topologies was assessed with Unique Tree v. 1.9, kindly provided by Thomas Wong and available upon request. Node support was estimated via nonparametric bootstrapping of 100 bootstraps replicates in IQ-TREE and mapped onto the ML tree with the best loglikelihood.

We further scanned all these lepidopteran protein sets for several gene families associated with detoxification function, namely P450 monooxygenases (P450s), carboxyl- and choline esterases (CCEs), UDP-glycosyltransferases (UGTs), glutathione Stransferases (GSTs), ATP-binding cassettes (ABCs). We identified the protein families of all proteins by running InterProScan $\mathrm{v}$. 5.36-75 (-appl Pfam -goterms; Jones et al. 2014); additionally, we ran a local BLASTP against the UniRef50 database (ftp.uniprot. org/pub/databases/uniprot/uniref/uniref50/uniref50.fasta.gz; 


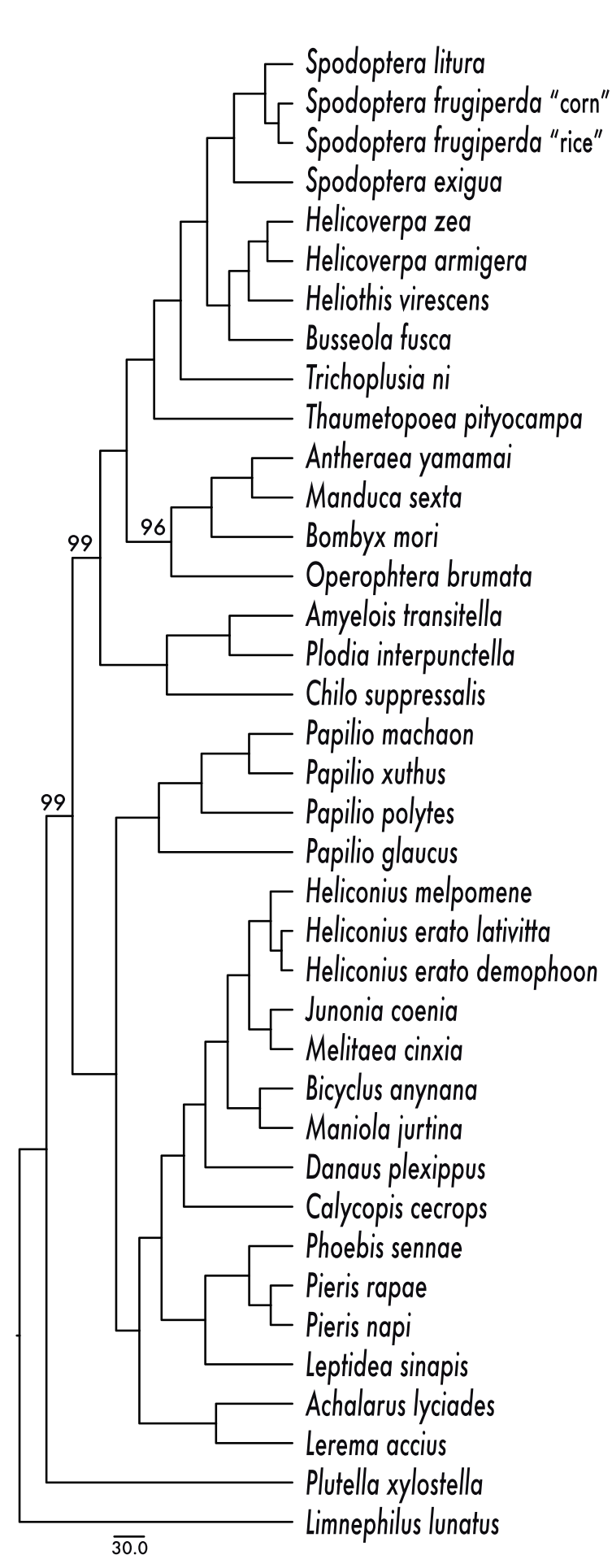

P450 CCE UGT GST ABC
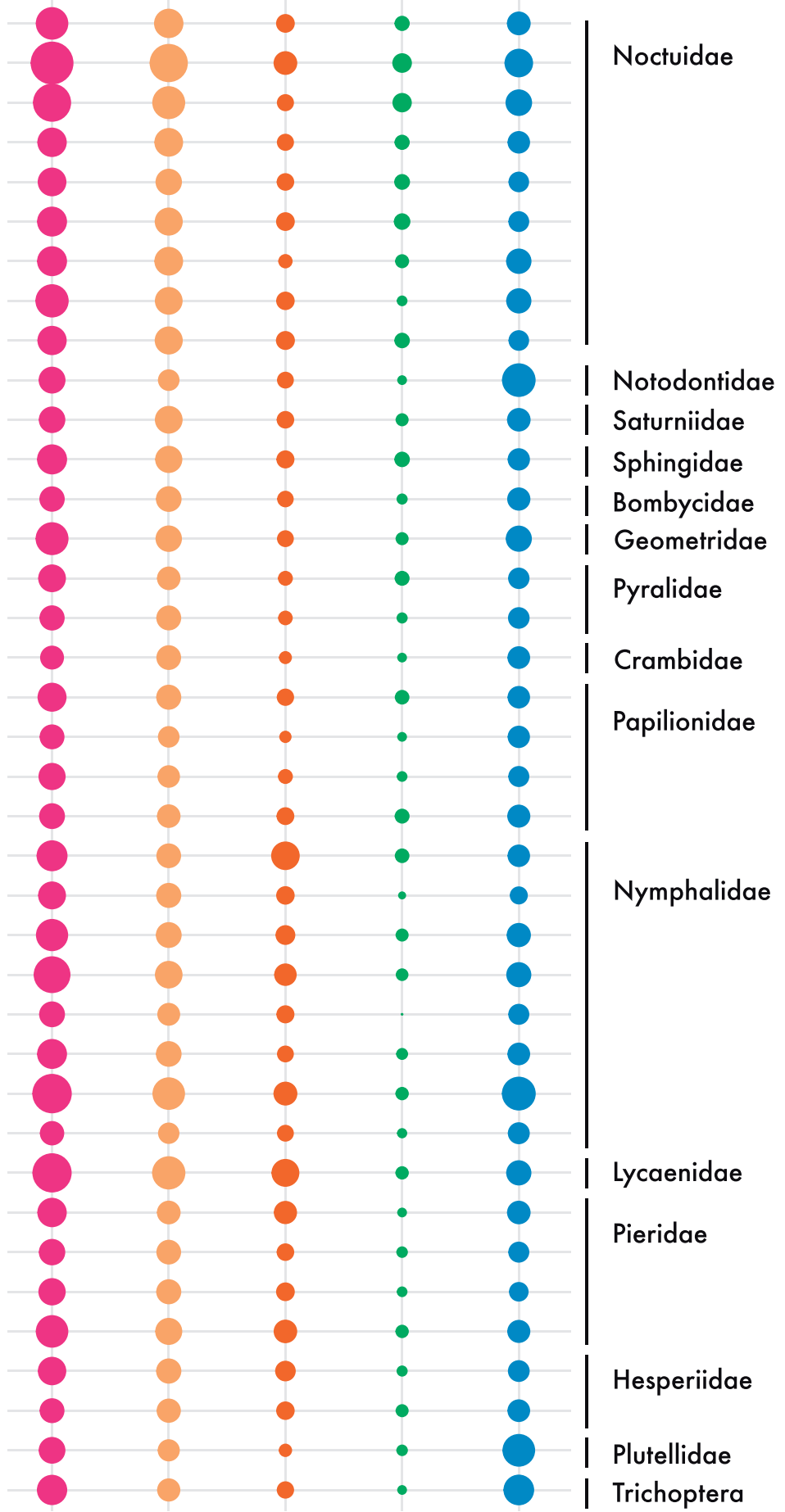

ymphalidae

255075100125150175200225

- 100000

Figure 4 Comparison of Lepidoptera genomes and inferred phylogenetic relationships. Shown is the ML phylogeny based on 1367 single-copy BUSCOs (left, all nodes have 100\% support unless otherwise noted). A number of detoxification gene members of five main detoxification families, P450s, CCEs, UGTs, GSTs, ABCs, are presented per species in a bubble plot generated with ggplot2.

release version July 31, 2019, accessed August 20, 2019) using an e-value cutoff of 1e-3. Based on these annotations, genes were selected to belong to any of the gene families of interest if it had a match to one of the Uniref50 cluster terms or Pfam- or
InterProScan identifiers (Supplementary Table S12). The number of detoxification gene members of the five main detoxification families was plotted for each species in a bubble plot generated with ggplot2 (Wickham 2016; Figure 4). 


\section{Comparative analysis of Spodoptera-specific genes}

We used OrthoFinder v. 2.3.11 using default settings (Emms and Kelly 2015) to identify homologs within the Spodoptera clade. We included the genome protein sequence files from three Spodoptera species: S. exigua (this study), S. litura [direct receival OGSv1 September 28, 2019 from authors (Cheng et al. 2017)] and S. frugiperda (ftp://ftp.cngb.org/pub/CNSA/CNP0000513/CNS0099235/ CNA0003276/Sf_20190612ynM_v1.pep, accessed September 20, 2019; Liu et al. 2019). In addition, we included five closely related but diverse Lepidoptera species: Heliothis virescens (ftp://ftp.ncbi. nlm.nih.gov/genomes/all/GCA/002/382/865/GCA_002382865.1_ K63_refined_pacbio/GCA_002382865.1_K63_refined_pacbio_pro tein.faa.gz, accessed September 20, 2019; Fritz et al. 2018), Helicoverpa zea (https://data.csiro.au/collections/\#collection/ CIcsiro:23812v3, accessed August 21, 2019; Pearce et al. 2017), Helicoverpa armigera (https://data.csiro.au/collections/\#collection/ CIcsiro:23812v3, accessed August 21, 2019; Pearce et al. 2017), Trichoplusia ni (ftp://www.tnibase.org/pub/tni/tni_protein_v1.fa. gz, accessed September 20, 2019; Chen et al. 2019), and Bombyx mori (http://silkbase.ab.a.u-tokyo.ac.jp/cgi-bin/download.cgi, accessed August 20, 2019; International Silkworm Genome Consortium 2008).

We identified 119 orthogroups (OGs) containing sequences only from the 3 Spodoptera species (Supplementary Table S13.1). Of these 119 OGs, only 7 OGs were DE in the larval stage (cluster 4, Supplementary Table S13.2). Of these seven OGs, three OGs were "uncharacterized" protein, and four OGS were annotated as: nuclear complex protein (OG0013351), REPAT46 (OG0014254), trypsin alkaline-c type protein (OG0014208), and mg7 (OG0014260; Supplementary Table S13.2) for which we performed gene tree analyses. For the gene tree analyses, we extended our dataset based on the original OrthoFinder run by including similar sequences from related species to additionally verify the lineage-specificity of these genes. Using the identified S. exigua sequences within the lineage-specific OGs as queries, we searched for close homologs using BLASTX (Bravo et al. 2019) against the NCBI protein database online (Sayers et al. 2020). Thus, the resulting datasets used to construct gene trees were compiled with some differences. The gene tree of nuclear pore complex proteins was composed of Spodoptera OG sequences and all Lepidoptera nuclear complex DDB_G0274915 proteins from the NCBI-nr database (accessed October 2, 2020, keyword “DDB_G0274915"). The initial BLAST identifications of Spodopteraspecific OG sequences showed high similarity with DDB_G0274915-like nuclear pore complex proteins. For the remaining three datasets, we additionally included clusters of homologous genes from OrthoDB v. 10 (Kriventseva et al. 2019). For the REPAT protein dataset, we added the ortholog cluster ("16151at7088") consisting of Multiprotein bridge factor 2 (MBF2) orthologs. MBF2 proteins are described to be homologs of REPAT genes in other Lepidoptera species, and have been therefore included (Navarro-Cerrillo et al. 2013). The REPAT protein gene tree dataset included all protein sequences from Navarro-Cerrillo et al. (2013). For a second REPAT tree, we only analyzed sequences from the BREPAT class (Navarro-Cerrillo et al. 2013). For both, the trypsin and mg7 gene tree datasets, we included clusters of homologous genes from OrthoDB v. 10 based on the linked cluster to our closest BLAST hit via the online NCBI protein database. For the trypsin gene tree dataset, we added the ortholog cluster "118933at50557" consisting of "serine protease" orthologs. These homologous sequences were selected because the S. litura sequence ("SWUS10076430") from the Spodoptera-specific OG formed a member of this group. All insect orthologs were included. Finally, the mg7 gene tree dataset included the ortholog group "15970at7088" from OrthoDB v. 10 (accessed September 15, 2020), because the S. litura sequence (“SWUS10113290") was an ortholog member. For a second tree, we included all genes derived from He et al. (2012), where the expression of mg7 in the midgut of S. litura was studied and homologs in related lepidopteran species were analyzed. Finally, we searched for potential paralogs of all target genes in the protein sets of S. exigua, S. litura, and S. frugiperda using BLASTP (max_hsps 1, best_hit_overhang 0.1 and Evalue cutoff $\leq 1 \mathrm{e}-5$ ) with NCBI-BLAST + v. 2.6.0 (Camacho et al. 2009) against a local BlastDB of above gene tree datasets of nuclear pore complex, REPAT, trypsin, and mg7 proteins.

For all genes, sequences were aligned using MAFFT v. 7.471 with the L-INS-i method and default settings (Katoh and Standley 2013). Gene trees were reconstructed using IQ-TREE v. 1.6.12 (Nguyen et al. 2015; Chernomor et al. 2016) using the ML method and implementing bootstrap with 100 replications. The preferred model was applied based on the model selection (Kalyaanamoorthy et al. 2017). For the nuclear pore complex gene tree, the best-fit model was "WAG+F+G4," for REPAT including both $\alpha$ REPAT and BREPAT proteins "WAG+F+R4," for the gene tree consisting only $\beta R E P A T$ proteins "VT $+G 4$," for the trypsin gene tree "WAG+F+R5" finally for both mg7 based gene trees "LG+G4." All gene alignment files are provided at the Dryad digital repository.

The gene trees were rooted dependent on included species and gene composition, aiming for earliest branching genes or species, for example, by selecting the earliest branching lineages from Kawahara et al. (2019). For the nuclear pore complex protein gene tree, Papilio xuthus was used for rooting since it branched early within Papilionidae (Kawahara et al. 2019). For the REPAT gene tree, we used the same approach as Navarro-Cerrillo et al. (2013), which rooted the tree using the REPAT-like27 and REPATlike28 cluster. However, for the limited REPAT gene tree only including BREPAT class genes, we rooted using group $V$ of the $\beta R E P A T$ class according to the first group branching off (NavarroCerrillo et al. 2013). The trypsin tree was rooted using the branch, giving rise to a Hymenoptera-specific cluster. Finally, the mg7 gene trees were rooted using either Choristoneura fumiferana (Tortricidae) (mg9 cluster) or, if absent, Amyelois (Pyralidae; Kawahara et al. 2019).

\section{Results \\ Genome annotation and comparison to other Lepidoptera genomes}

The total size of the final polished assembled genome was $419 \mathrm{Mb}$, which was divided over 946 contigs (largest contig = $4.15 \mathrm{Mb}$ ) with $\mathrm{N} 50=1.1 \mathrm{Mb}$ (Table 1 ). To confirm the assembly genome size, a k-mer counting approach was used. After counting the 21 and 27 mers in the Illumina dataset, the count tables were analyzed with GenomeScope. The genome size as estimated by kmer counting was $\sim 370 \mathrm{Mb}$, which correlated with the Nanopore assembly size (which is slightly larger). The genome size of S. exigua presented here, as well as the GC content (given in \%), is comparable with other published Spodoptera genomes and the preprint version of the S. exigua genome (Zhang et al. 2020; Table 1).

The BUSCO (v. 3) assessments indicated that the quality and completeness of our de novo assembly was good (complete: $96.8 \%$; fragmented: $1.0 \%$; missing: $2.2 \%$ ) and comparable with other 
Table 1 Genome metrics of Spodoptera exigua and other published Spodoptera genomes

\begin{tabular}{|c|c|c|c|c|c|c|c|c|c|}
\hline \multicolumn{2}{|l|}{ Species } & \multicolumn{2}{|c|}{ Sequencing information } & Reference & \multicolumn{2}{|l|}{ Genome assembly } & No. of contigs & GC content & Protein \\
\hline $\begin{array}{l}\text { Spodoptera } \\
\text { exigua }\end{array}$ & & $\begin{array}{r}\text { Female } \\
\text { pupa }\end{array}$ & $\begin{array}{l}\text { PacBio }+ \\
\text { Illumina } \\
+ \text { Hi-C }\end{array}$ & $\begin{array}{l}\text { Zhang et al. } \\
\text { (2020) }\end{array}$ & $446.8 \mathrm{Mb}$ & $3.5 \mathrm{Mb}$ & 667 & 36.67 & 17,727 \\
\hline $\begin{array}{l}\text { Spodoptera } \\
\text { litura }\end{array}$ & & Male adults & Illumina & $\begin{array}{c}\text { Cheng et al. } \\
\text { (2017) }\end{array}$ & $438.3 \mathrm{Mb}$ & $0.068 \mathrm{Mb}$ & 13,636 & 37 & 15,317 \\
\hline $\begin{array}{l}\text { Spodoptera } \\
\text { frugiperda }\end{array}$ & & $\begin{array}{l}\text { Sf21 cell } \\
\text { line }\end{array}$ & Illumina & $\begin{array}{l}\text { Kakumani } \\
\text { et al. (2014) }\end{array}$ & $358.0 \mathrm{Mb}$ & $0.008 \mathrm{Mb}$ & 97,607 & 32.97 & 11,595 \\
\hline $\begin{array}{l}\text { Spodoptera } \\
\text { frugiperda }\end{array}$ & "corn" & $\begin{array}{l}\text { Two male } \\
\text { larvae }\end{array}$ & Illumina & $\begin{array}{c}\text { Gouin et al. } \\
(2017)\end{array}$ & $437.9 \mathrm{Mb}$ & $21.6 \mathrm{~Kb}$ & - & 36 & 21,700 \\
\hline $\begin{array}{l}\text { Spodoptera } \\
\text { frugiperda }\end{array}$ & "rice" & $\begin{array}{l}\text { Single male } \\
\text { larva }\end{array}$ & Illumina & $\begin{array}{l}\text { Gouin et al. } \\
\text { (2017) }\end{array}$ & $371.0 \mathrm{Mb}$ & $25.4 \mathrm{~Kb}$ & - & 36 & 26,329 \\
\hline $\begin{array}{l}\text { Spodoptera } \\
\text { frugiperda }\end{array}$ & "rice" & $\begin{array}{l}\text { Single male } \\
\text { larva }\end{array}$ & $\begin{array}{l}\text { PacBio }+ \\
\text { Illumina } \\
+ \text { Hi-C }\end{array}$ & $\begin{array}{c}\text { Gimenez et } \\
\text { al. (2020), } \\
\text { Nam et al. } \\
(2020)\end{array}$ & $379.9 \mathrm{Mb}$ & - & - & 36.37 & 22,026 \\
\hline $\begin{array}{l}\text { Spodoptera } \\
\text { frugiperda }\end{array}$ & & $\begin{array}{l}\text { Single male } \\
\text { adult }\end{array}$ & $\begin{array}{l}\text { MGISEQ + } \\
\text { Hi-C }\end{array}$ & $\begin{array}{l}\text { Gui et al. } \\
(2020)\end{array}$ & $543.7 \mathrm{Mb}$ & $0.09 \mathrm{Mb}$ & 29,584 & 36.52 & 22,201 \\
\hline $\begin{array}{l}\text { Spodoptera } \\
\text { frugiperda }\end{array}$ & & $\begin{array}{l}\text { Single male } \\
\text { adult }\end{array}$ & $\begin{array}{l}\text { PacBio }+ \\
\text { Illumina } \\
+ \text { Hi-C }\end{array}$ & $\begin{array}{l}\text { Zhang et al. } \\
\text { (2020) }\end{array}$ & $390.4 \mathrm{Mb}$ & $5.6 \mathrm{Mb}$ & 776 & 36.4 & 22,260 \\
\hline $\begin{array}{l}\text { Spodoptera } \\
\text { frugiperda }\end{array}$ & & $\begin{array}{r}\text { Female } \\
\text { pupa }\end{array}$ & $\begin{array}{c}\mathrm{PacBiO}+ \\
\mathrm{Hi}-\mathrm{C}\end{array}$ & $\begin{array}{l}\text { Xiao et al. } \\
\text { (2020) }\end{array}$ & $486.3 \mathrm{Mb}$ & $1.1 \mathrm{Mb}$ & 618 & 36.4 & 22,623 \\
\hline
\end{tabular}

lepidopteran genomes (Supplementary Figure S3). By these quality metrics, the S. exigua assembly is comparable with those of fellow lepidopterans, facilitating comparative genomic analyses.

Using our final assembly, an OGS was generated by automatic annotation and transcriptomic RNA-seq datasets of $18 \mathrm{~S}$. exigua samples (see below) as supporting evidence. The OGS (v. 1.1), consists of 18,477 proteins and is provided at the Dryad digital repository.

\section{Gene expression analyses across the whole life- cycle of Spodoptera exigua}

The major developmental stages across the whole life-cycle of S. exigua, namely embryonic stage (egg), early first-instar larva, early third-instar larva, pupa, and adult (both sexes: female and male), were sequenced on an Illumina NovaSeq 6000 system at an average of 13.4 million $\mathrm{PE} 2 x 150$ nt reads (6.9-22.5 million reads per sample; Supplementary Table S1.3). Based on these reads, we performed differential expression analyses using our de novo assembled S. exigua genome as a reference.

We first compared gene expression from subsequent different developmental stages and sexes based on pairwise comparisons to determine the dynamic changes in gene expression during development. A striking number of significantly DE transcripts ( $n=4974$ transcripts) was detected during early embryonic development (between the embryonic and the first-instar larval stage; Figure 1). Notably, this rapid change in the expression dynamics of S. exigua was the largest during the entire life cycle (Figure 1 and Supplementary Table S14). In contrast, the smallest change in gene expression was between first- and third-instar larvae
( $n=1222$ transcripts). A larger change in gene expression was also observed between pupa and male adult ( $n=3112$ transcripts) compared with pupa to female adult ( $n=2061$ transcripts), likely due to the fact that female pupae were analyzed. For an overview of relationships between the different life stages based on identified significant changes in gene expression see Supplementary Figure S4. Supplementary Table S15 provides an overview of all DE genes identified per pairwise comparison of the developmental stages.

We further identified 9896 transcripts as DE across all pairwise comparisons. Hierarchical clustering revealed 14 clusters of DE transcripts with similar expression patterns (Figure 2). Of these, the gene expression of eight clusters could be associated with a single developmental stage or similar subsequent developmental stages, for example, one cluster for the larval stage (see also Supplementary Figure S2). For these eight clusters, statistically overrepresented GO terms were identified using FDR-adjusted Pvalue $(<0.05)$ and were further summarized to generic GO slim categories (Figure 3).

For the embryonic stage (cluster 11, Figure 3), there was an enrichment of GO categories associated with ribosome biogenesis (GO:0042254), ribonucleoprotein complex assembly (GO:0022618), transfer RNA (tRNA) metabolic process (GO:0006399), translation (GO:0006412), and cell cycle (GO:0007049). The enrichment of these categories highlights the rapid succession of cell cycles associated with chromatin replication and initiation of transcription and translation for embryo patterning (Koutsos et al. 2007). Detailed investigation of DEs gene annotations based on the Arthropoda database (Supplementary Tables S4 and S5) revealed 
several known genes important in morphogenesis, for example, during the embryonic stage Krüppel-like transcription factors (Kaczynski et al. 2003; McCulloch and Koenig 2020), specificity proteins (Kennedy et al. 2016), and several WD-repeat containing proteins (Smith 2008).

We did not identify a specific cluster for the first larval stage nor for the third larval stage, but rather one cluster including both larval stages (=larval stage cluster, cluster 4, Figure 3). The larval stage was enriched for genes involved in general metabolic processes, such as signal transduction (GO:0007165), biosynthetic processes (GO:0009058), and secondary metabolic processes (GO:0019748). Several genes having a key role in the digestion of plant material and herbivore success were significantly DE within the larval stage (see Supplementary Table S4). These include REPAT genes (Herrero et al. 2007; Navarro-Cerrillo et al. 2013), trypsins (Muhlia-Almazán et al. 2008), cuticle proteins (Celorio-Mancera et al. 2013; Müller et al. 2017; Orsucci et al. 2018; Breeschoten et al. 2019), and members of prominent detoxification gene families such as cytochrome P450s (P450), carboxyl/cholinesterases (CCEs), GST, and UGT. The pupal stage varied from the larval stage in that there was significant enrichment in processes associated with cell differentiation (GO:030154), anatomical structure formation involved in morphogenesis (GO:0048646), and anatomical structure development (GO:0048856).

We further identified several pupal cuticle proteins as significantly DE within this pupal stage. The female adult stage (cluster 12) was enriched for genes involved in for example, cell cycle (GO:0007049), chromosome segregation (GO:0007059) and chromosome organization (GO:0051276), anatomical structure development (GO:0048856), and biosynthetic process (GO:0009058) and we identified orthologs of several homeotic genes(-like), such as Bicaudal C, Sex combs reduced, and proboscipedia. For the male adult stage (cluster 2, Figure 3), there was an enrichment of GO categories associated with for example, mRNA processing (GO:0006397), cellular aa metabolic process (GO:0006520), cellular component assembly (GO:0022607), and biosynthetic process (GO:0009058). For the female and the male adult stage, we further identified several sex-specific genes as DE, such as vitellogenin and vitellogenin receptor in the female (Rotllant et al. 2017) and testisspecific serine/threonine-protein kinase 2 (Kim et al. 2019) or ejaculatory bulb-specific protein (Liu et al. 2020) in the male stage, respectively. One cluster (cluster 14) was specific for both adult sexes but was enriched only for the carbohydrate metabolic process (GO:0005975). In contrast, cluster 9 (comprised of the pupa and both adult sexes) was enriched for several GO categories: cellular aa metabolic process (GO:0006520), catabolic process (GO:0009056), biosynthetic process (GO:0009058), and cellular nitrogen compound metabolic process (GO:0034641; see Figure 3 and Supplementary Table S10).

\section{Lepidopteran phylogenomics and detoxification gene content evolution}

The phylogenomic analysis correctly placed S. exigua within the Spodoptera clade and as the sister-group to the clade containing S. litura and S. frugiperda (Figure 4; Le Ru et al. 2018; Kergoat et al. 2021). In addition, the inferred species relationships within Lepidoptera were in agreement with previous findings (Kawahara et al. 2019). We further scanned all lepidopteran genomes for gene families associated with detoxification functions. This included: gene families involved in phase I of the detoxification pathway such as cytochrome P450 and CCE (Kant et al. 2015); gene families involved in phase II, such as UGT and GST; and the gene family ABC involved in phase III (Li et al. 2007; Heidel-
Fischer and Vogel 2015; Kant et al. 2015). Based on the annotation of the lepidopteran genomes, we searched for expanded detoxification-related genes (Figure 4 and Supplementary Table S16). Expansion of major genes families involved in detoxification was mainly visible for S. frugiperda ("corn" strain) within the Noctuidae. In the following, we analyzed in greater detail several lineage-specific genes.

\section{Potential lineage- and stage-specific candidate genes as targets for pest-control}

We used OrthoFinder v. 2.3.11 (Emms and Kelly 2015) to identify homologous gene sequences in the genomes of eight closely related but diverse lepidopteran species, including three Spodoptera species, S. exigua, S. litura, and S. frugiperda. We aimed to identify Spodoptera-specific OGs, as such lineage-specific genes would be candidates for targeted pest-outbreak management development. We identified in total 119 OGs containing genes from only the three Spodoptera species (Supplementary Table S13.1).

Since the larval feeding stage of Spodoptera is the most detrimental to crops, we further selected seven OGs for which the S. exigua gene representative is DE in the larval stage cluster (cluster 4). For three of the seven genes, the closest homologs were "uncharacterized" proteins (Supplementary Table S13.2). The four remaining genes were annotated as: nuclear complex protein (OG0013351), REPAT46 (OG0014254), trypsin alkaline-c type protein (OG0014208), and mg7 (OG0014260; Supplementary Table S13.2). We confirmed the expression of all seven genes by checking the number of RNA-Seq reads mapped to each assembled transcript based on the results of the transcript abundance estimation with RSEM. The read count in the larval stages (first and third larval stages) was higher than in the other stages (Supplementary Table S17). Several reads derived from other stages mapped to the protein sequences, but the number of these mapped reads was low (Supplementary Table S17).

For the four putative lineage- and stage-specific annotated genes, we validated their Spodoptera-specificity by constructing gene trees of Spodoptera sequences with their most similar sequences identified from other lepidopteran species. We confirmed Spodoptera-specificity when all Spodoptera sequences in the gene tree reconstruction clustered together in a monophyletic group. For two of the annotated genes (mg7 and REPAT), we constructed two different gene trees. These gene trees were built on two different datasets (extended and reduced). The identification of putative homologs in related species varied per gene as well as the number of included sequences and species for the gene tree analyses [nuclear complex protein (OG0013351): 20 sequences, 3494 aa positions, REPAT46 (OG0014254) extended dataset containing both $\alpha$ REPAT and $\beta R E P A T$ clusters: 153 sequences, 863 aa positions, reduced dataset containing only the BREPAT cluster: 91 sequences, 717 aa positions, trypsin alkaline-c type protein (OG0014208): 69 sequences, 1101 aa positions, and mg7 (OG0014260): extended dataset: 27 sequences, 368 aa positions, reduced dataset: 17 sequences, 350 aa positions].

The gene tree of the nuclear pore complex proteins showed that the Spodoptera-specific genes form a single cluster, nested within lepidopteran DDB_G0274915-like nuclear pore complex proteins and sister to Helicoverpa sequences (Supplementary Figure S5). The reduced mg7 dataset comprised sequences from the Spodoptera-specific OG in addition to the ortholog group "15970at7088" from OrthoDB. For the extended mg7 dataset, we additionally included all "mg" protein sequences according to $\mathrm{He}$ et al. (2012). The ortholog group "15970at7088" included nine single-copy genes present in other butterfly species and we found 
two paralogous copies in S. litura, likely due to a specific gene duplication. In order to evaluate whether other paralogs were present in any of the Spodoptera gene sets, we blasted the protein sequences against a local blast database of $\mathrm{mg} 7$ sequences comprising the sequences from OrthoDB, OG0014260, and He et al. (2012). In S. exigua, we identified three paralogs, which according to the GFF file are located (mRNAs) consecutively on the genome: 1268792-1275628, 1276053-1279376, 1280841-1286731. Similarly, in S. litura, we identified two and three paralogs in S. frugiperda. To test if the existence of multiple paralogs for mg7 is specific for Spodoptera, we analyzed the protein sets of five related Lepidoptera species as used in the initial OrthoFinder run. Running the same blast searches but using the protein sets of $B$. mori, $H$. armigera, H. zea, $H$. virescens, and T. ni all detected a single gene copy with reliable BLAST scores. Both the reduced and the extended mg7 gene trees included all identified Spodoptera paralogs. The reduced mg7 gene tree including all paralog Spodoptera genes and the single-copy homologs from OrthoDB showed that Spodoptera-specific OG sequences were clustered together (Supplementary Figure S6). This cluster formed a sister clade to all remaining Spodoptera paralogs and the H. armigera gene. In the extended mg7 gene tree, the Spodoptera-specific OG sequences did not form a monophyletic clade but did cluster together with the mg7 genes of C. fumiferana, H. armigera, and S. litura derived from He et al. (2012) (Supplementary Figure S7).

For the REPAT gene analyses, we compiled two datasets. Both datasets consisted of sequences derived from the Spodoptera-specific OG, the MBF2 ortholog group "16151at7088" from OrthoDB and all protein sequences according to Navarro-Cerrillo et al. (2013). The reduced dataset only contained protein sequences belonging to the $\beta$ REPAT class, whereas the extended dataset included both aREPAT and $\beta$ REPAT classes. In both gene tree analyses, the Spodoptera-specific OG sequences clustered together with the annotated REPAT46 gene from S. exigua (Supplementary Figures S8 and S9). The Spodoptera-specific OG is placed in the BREPAT cluster, sensu Navarro-Cerrillo et al. (2013), where it is placed within group VI (Navarro-Cerrillo et al. 2013). Further, in total 54 putative REPAT proteins have been identified in the S. exigua protein set which were included in both gene tree datasets (Supplementary Table S18).

The gene tree of the trypsin proteins showed a monophyletic clustering of all Lepidoptera-derived trypsin genes (Supplementary Figure S10). In addition, all Spodoptera trypsins were clustered within one monophyletic clade, with the Spodoptera-specific OG nested within. Trypsins occurred in all Lepidoptera species in large numbers, thus we compared various OrthoFinder runs under different stringency settings [varying the inflation parameter from 1, 1.2, 1.5 (default), 3.1, and 5] to test the degree of "Spodoptera-specificity" of this OG. In all five runs, the OG containing the Spodoptera trypsin genes was stable (e.g., lineage-specific) and remained unchanged.

\section{Discussion}

Using a combination of Oxford Nanopore long-read data and Illumina short-read data for the genome sequencing approach, we generated a high-quality genome and transcriptome of the beet armyworm, S. exigua. These resources will be beneficial for future research on S. exigua and other noctuid pest species. The developmental gene expression profile of S. exigua demonstrated that the transition from embryo to larva is the most dynamic period of the beet armyworm's transcriptional activity. Within the larval stage the transcriptional activity was highly similar between early (first) and late (third) instars, making the early larval stage an ideal stage for pest-control (see below). Genes involved in the secondary metabolic process (GO:0019748) were only expressed in the larval stages (Figure 3). In addition, several prominent genes involved in digestion and detoxification, including cytochrome P450s and UGTs, and potential target genes for pest control could be identified which are specifically expressed in the larval stage (Supplementary Table S4).

The significant enrichment in the pupal stage in processes associated with anatomical structure development reflects the dramatic structural changes of the larva to the adult (Truman and Riddiford 2019). The identified pupal cuticle proteins within the pupal stage have been reported previously by other studies and reflect the morphological changes in wing disc and the larva-topupa metamorphosis (Gu et al. 2013; Ou et al. 2014).

The gene expression analyses of the developmental transcriptome of S. exigua revealed larval stage-specific upregulated genes (cluster 4, Figures 2 and 3). These identified genes are strong candidates for targeted RNAi of feeding larvae. Targeted RNAi of genes involved in vital functions of the most important larval stage can be an efficient strategy to minimize the detrimental effect of pest species (Xue et al. 2012). The larva stages of Noctuidae insects are the most damaging to plants. Our homology search revealed seven potential Spodoptera-specific genes with upregulation in the first- and third-instar larval stages, and highest expression levels in the third-instar stage (Supplementary Table S17). Four of these seven genes were annotated and we confirmed for three of them Spodoptera-specificity by gene tree analyses.

One putative Spodoptera-specific OG consisted of nuclear pore complex proteins. These proteins are involved in the transport of particles through the nuclear envelope (Alber et al. 2007). Although the gene tree did not follow well-established lepidopteran relationships (Kawahara et al. 2019), for example, Noctuoidea nested within Papilionoidea (Supplementary Figure S5), all identified Spodoptera nuclear pore complex proteins clustered together. This is a prerequisite for potential target genes, showing a clear separation of Spodoptera-derived sequences to sequences of other species.

We identified mg7 as a potential target gene for RNAi. This gene was previously reported to be highly upregulated in all larval stages in the midgut of S. litura with an expression peak after larvae have molted into the sixth larval stage (He et al. 2012). Our results show a similar pattern with an increased expression towards the third-instar larva (Supplementary Table S17). Expression in the midgut suggests a role in digestion-related processes (He et al. 2012). Only the gene tree based on the reduced dataset showed clustering of Spodoptera-specific mg7 genes (Supplementary Figure S6). He et al. (2012) reported several homologs, mg2, mg7, mg9, and mg17 in related species which we included in the extended gene tree reconstruction (Supplementary Figure S7). The genes derived from the Spodoptera-specific OG form a monophyletic group with the $\mathrm{mg} 7$ genes of $\mathrm{C}$. fumiferana, H. armigera, and S. litura derived from He et al. (2012), establishing orthology of Noctuidae and Tortricidae sequences and consequently challenging the Spodoptera-specificity for this candidate gene. The spruce budworm, C. fumiferana is a notorious coniferfeeding pest restricted to the Nearctic region where it is considered one of the most destructive insect defoliators (Lumley and Sperling 2010; Volney and Fleming 2007). The extended phylogeny identified homologous clusters (although with low support values) of "mg" genes (mg7, mg17, and mg9) in related lepidopteran species. The close relationship of additional gene family members from other lepidoptera makes mg7 more a potential 
candidate for RNAi-based pest-formation control in a wider range of lepidopteran pest species with the caveat that more work is needed to resolve lineage- and/or Spodoptera-specificity.

Finally, a strong potential target gene for biocontrol are the aREPAT proteins which are involved in various physiological processes and can be induced in response to infections, bacterial toxins and other microbial pathogens within the larval midgut (Herrero et al. 2007; Navarro-Cerrillo et al. 2013). Upregulation of REPAT genes has been identified in response to the entomopathogenic Bacillus thuringiensis (Herrero et al. 2007). In S. frugiperda, REPAT genes were associated with defense functions in other tissues than the midgut and found to be likely functionally diverse with roles in cell envelope structure, energy metabolism, transport, and binding (Machado et al. 2016).

REPAT genes are divided in two classes based on conserved domains. Homologous genes of the aREPAT class are identified in closely related Spodoptera and Mamestra species, whereas $\beta R E P A T$ class homologs are identified in distantly related species, for example, HMG176 in H. armigera and MBF2 in B. mori (NavarroCerrillo et al. 2013). Our analyses found that REPAT genes (and homologs like MBF2 members) from distantly related species are nested within the $\beta$ REPAT cluster, while the $\alpha$ REPAT class is exclusive for Spodoptera and very closely related species like Mamestra spp. (Navarro-Cerrillo et al. 2013; Zhou et al. 2016; Supplementary Figures S8 and S9). In contrast to NavarroCerrillo et al. (2013) where $\alpha$ REPAT and $\beta$ REPAT form sister clades, our tree topology show $\alpha$ REPAT genes to be nested within BREPAT.

Previously, 46 REPAT genes were reported for S. exigua (Navarro-Cerrillo et al. 2013), while we detected 54 REPAT genes in the S. exigua genome (Supplementary Table S18). The genes of S. exigua, S. litura, and S. frugiperda from the Spodoptera-specific OG as identified here cluster together with REPAT46 from S. exigua and thus are group VI $\beta$ REPAT genes (Supplementary Figure S8). As shown in Navarro-Cerrillo et al. (2013) and here (Supplementary Figure S8), group VI $\beta$ REPATs are comprised of Spodoptera- and other noctuid-derived genes, like Helicoverpa and Mamestra. The Noctuidae family is one of the most damaging groups of pests to agriculture, which is recognized by naming of a "pest clade" where species from the genera Spodoptera, Helicoverpa, and Mamestra are included (Mitchell et al. 2006; Regier et al. 2017). Overall, the results presented here show that REPAT gene members of especially the $\alpha$ REPAT class and the group VI $\beta$ REPATs are putatively promising candidates for targeted RNAi in notorious pest species belonging to Spodoptera and closely related genera in Noctuidae, given their Spodoptera- and/or Noctuidae-specificity.

\section{Conclusions}

The genome and developmental transcriptome including all major stages: embryonic, larval, pupal, and adult stages of both sexes, of the beet armyworm S. exigua provides a valuable genomic resource for this important pest species. Using a dual sequencing approach including long- and short-read data, we were able to provide a genome that is comparable to fellow lepidopterans, strongly supporting the use of these resources in further genome comparisons. Based on the differential gene expression analyses, we identified developmental stage-specific (embryonic, larva, pupa, or adult) or sex-specific (female, male adult) transcriptional profiles. Of particular interest are the identified genes specifically upregulated in the larval stages because those stages are most detrimental to the host plants. We have further validated these larva-specific genes for their suitability for RNAibased targeted pest control by comparative genome analyses. RNAi-mediated insect control can be a powerful tool if selected target gene(s) are essential genes in insect tissues to trigger toxic effects. In addition, the target gene(s) should be pest speciesspecific or specific to a range of closely related pest species and should not harm nontarget organisms. In this context, Spodoptera lineage-specific target gene(s) are of high interest due to the high number of notorious pest species in this genus causing enormous agricultural damage resulting in economic losses worldwide. Analyzing the homologous relationships of the identified potential target genes and including a broad selection of other insect species allowed us to verify the specificity of three candidate genes for the genus Spodoptera and one candidate for RNAi-based pest-formation control in a wider range of lepidopteran pest species. Additional in-depth research may further confirm the cladespecificity of these genes and their potential application in RNAimediated pest-outbreak management.

\section{Data availability}

The final genome assembly was submitted to the NCBI GenBank database and is available under the BioProject PRJNA623582, accession JACEFF000000000, version JACEFF010000000 is used in this study. All raw reads from the Illumina, MinION, and PromethION sequencing runs and Illumina RNA-Seq run were submitted to the NCBI SRA database under accession number PRJNA623582.

Supplemental material available at figshare: https://doi.org/ 10.25387/g3.14995326.

Further genome datasets and other datasets generated during the current study are provided at the Dryad digital repository https://doi.org/10.5061/dryad.280gb5mq6.

\section{Acknowledgments}

We thank Els Roode and the late Hanke Bloksma for help with the S. exigua rearing and sample collection. We also thank Corné van der Linden for providing S. exigua photographs. We thank Entocare for their support to this project.

V.I.D.R. and S.S. initiated the study. V.I.D.R. collected samples. H.H.J. and R.P.D. performed genome and transcriptome sequencing, de novo assembly and automated annotation. T.B., S.S., and M.E.S. further optimized the assembly and annotation and performed differential gene expression, comparative genome, and gene tree analyses. S.S., V.I.D.R., T.B., and M.E.S. wrote the article. All authors read and approved the final article.

\section{Funding}

This project was funded by an Enabling Technologies Hotel grant from the Netherlands Organization for Health Research and Development (ZonMW) (project number 40-43500-98-4064). V.I.D.R. is supported by a VIDI-grant of the Dutch Research Council (NWO; VI.Vidi.192.041).

\section{Conflicts of interest}

The authors declare that there is no conflict of interest. 


\section{Literature cited}

Alber F, Dokudovskaya S, Veenhoff LM, Zhang W, Kipper J, et al. 2007. The molecular architecture of the nuclear pore complex. Nature. 450:695-701.

Andrews S. 2010. FastQC: A Quality Control Tool for High Throughput Sequence Data [Online]. Available online at: http:// www.bioinformatics.babraham.ac.uk/projects/fastqc/

Bi HL, Xu J, Tan AJ, Huang YP. 2016. Crispr/cas9-mediated targeted gene mutagenesis in Spodoptera litura. Insect Sci. 23:469-477.

Bolger AM, Lohse M, Usadel B. 2014. Trimmomatic: a flexible trimmer for illumina sequence data. Bioinformatics. 30:2114-2120.

Bravo GA, Antonelli A, Bacon CD, Bartoszek K, Blom MP, et al. 2019. Embracing heterogeneity: coalescing the tree of life and the future of phylogenomics. PeerJ. 7:e6399.

Breeschoten T, Ros VI, Schranz ME, Simon S. 2019. An influential meal: host plant dependent transcriptional variation in the beet armyworm, Spodoptera exigua (Lepidoptera: Noctuidae). BMC Genomics. 20:845.

Burand JP, Hunter WB. 2013. RNAi: future in insect management. J Invertebr Pathol. 112(Suppl):S68-74.

Camacho C, Coulouris G, Avagyan V, Ma N, Papadopoulos J, et al. 2009. Blast+: architecture and applications. BMC Bioinformatics. 10:421.

Celorio-Mancera MP, Wheat CW, Vogel H, Söderlind L, Janz N, et al. 2013. Mechanisms of macroevolution: polyphagous plasticity in butterfly larvae revealed by RNA-seq. Mol Ecol. 22:4884-4895.

Chen W, Yang X, Tetreau G, Song X, Coutu C, et al. 2019. A high-quality chromosome-level genome assembly of a generalist herbivore, Trichoplusia ni. Mol Ecol Resour. 19:485-496.

Cheng T, Wu J, Wu Y, Chilukuri RV, Huang L, et al. 2017. Genomic adaptation to polyphagy and insecticides in a major East Asian noctuid pest. Nat Ecol Evol. 1:1747-1756.

Chernomor O, von Haeseler A, Minh BQ. 2016. Terrace aware data structure for phylogenomic inference from supermatrices. Syst Biol. 65:997-1008.

International Silkworm Genome Consortium. 2008. The genome of a lepidopteran model insect, the silkworm Bombyx mori. Insect Biochem Mol Biol. 38:1036-1045.

Emms DM, Kelly S. 2015. Orthofinder: solving fundamental biases in whole genome comparisons dramatically improves orthogroup inference accuracy. Genome Biol. 16:157

EPPO. 2017. Eppo global pest database. https://gd.eppo.int/

Falcon S, Gentleman R. 2007. Using gostats to test gene lists for go term association. Bioinformatics. 23:257-258.

Fritz ML, DeYonke AM, Papanicolaou A, Micinski S, Westbrook J, Gould F. 2018. Contemporary evolution of a Lepidopteran species, Heliothis virescens, in response to modern agricultural practices. Mol Ecol. 27:167-181.

Fu X, Feng H, Liu Z, Wu K. 2017. Trans-regional migration of the beet armyworm, Spodoptera exigua (lepidoptera: Noctuidae), in north-east Asia. PLoS One. 12:e0183582.

Gimenez S, Abdelgaffar H, Goff GL et al. 2020. Adaptation by copy number variation increases insecticide resistance in the fall armyworm. Commun Biol. 3:664. https://doi.org/10.1038/s42003-020-01382-6

Goergen G, Kumar PL, Sankung SB, Togola A, Tamò M. 2016. First report of outbreaks of the fall armyworm Spodoptera frugiperda (J.E. Smith) (lepidoptera, noctuidae), a new alien invasive pest in west and central Africa. PLoS One. 11:e0165632.

Goodwin S, Gurtowski J, Ethe-Sayers S, Deshpande P, Schatz MC, et al. 2015. Oxford nanopore sequencing, hybrid error correction, and de novo assembly of a eukaryotic genome. Genome Res. 25: $1750-1756$
Gouin A, Bretaudeau A, Nam K, Gimenez S, Aury J-M, et al. 2017. Two genomes of highly polyphagous lepidopteran pests (Spodoptera frugiperda, noctuidae) with different host-plant ranges. Sci Rep. 7: 11816

Grabherr MG, Haas BJ, Yassour M, Levin JZ, Thompson DA, et al. 2011. Full-length transcriptome assembly from RNA-seq data without a reference genome. Nat Biotechnol. 29:644-652.

Gu J, Huang LX, Gong YJ, Zheng SC, Liu L, Huang LH, et al. 2013. De novo characterization of transcriptome and gene expression dynamics in epidermis during the larval-pupal metamorphosis of common cutworm. Insect Biochem Mol Biol. 43:794-808.

Gu X, Fu YX, Li WH. 1995. Maximum likelihood estimation of the heterogeneity of substitution rate among nucleotide sites. Mol Biol Evol. 12:546-557.

Gui F, Lan, T, Zhao, Y. et al. 2020. Genomic and transcriptomic analysis unveils population evolution and development of pesticide resistance in fall armyworm Spodoptera frugiperda. Protein Cell. https://doi.org/10.1007/s13238-020-00795-7.

Gimenez S, Abdelgaffar H, Goff, GL. et al. 2020. Adaptation by copy number variation increases insecticide resistance in the fall armyworm. Commun Biol. 3:664. https://doi.org/10.1038/s42003020-01382-6.

He W-Y, Rao Z-C, Zhou D-H, Zheng S-C, Xu W-H, et al. 2012. Analysis of expressed sequence tags and characterization of a novel gene, slmg7, in the midgut of the common cutworm, Spodoptera litura. PLoS One. 7:e33621.

Heidel-Fischer HM, Vogel H. 2015. Molecular mechanisms of insect adaptation to plant secondary compounds. Curr Opin Insect Sci. 8:8-14.

Herrero S, Ansems M, Van Oers MM, Vlak JM, Bakker PL, et al. 2007. Repat, a new family of proteins induced by bacterial toxins and baculovirus infection in Spodoptera exigua. Insect Biochem Mol Biol. 37:1109-1118.

Hu B, Huang H, Hu S, Ren M, Wei Q, et al. 2021. Changes in both trans- and cis-regulatory elements mediate insecticide resistance in a lepidopteron pest, Spodoptera exigua. PLoS Genet. 17: e1009403.

Huang JM, Zhao YX, Sun H, Ni H, Liu C, et al. 2021. Monitoring and mechanisms of insecticide resistance in Spodoptera exigua (Lepidoptera: Noctuidae), with special reference to diamides. Pestic Biochem Physiol. 174:104831.

Hurvich CM, Tsai CL. 1989. Regression and time-series model selection in small samples. Biometrika. 76:297-307.

Jansen HJ, Liem M, Jong-Raadsen SA, Dufour S, Weltzien FA, et al. 2017. Rapid de novo assembly of the European eel genome from nanopore sequencing reads. Sci Rep. 7:7213.

Jones DT, Taylor WR, Thornton JM. 1992. The rapid generation of mutation data matrices from protein sequences. Comput Appl Biosci. 8:275-282.

Jones P, Binns D, Chang H-Y, Fraser M, Li W, et al. 2014. Interproscan 5: genome-scale protein function classification. Bioinformatics. 30:1236-1240.

Kaczynski J, Cook T, Urrutia R. 2003. Sp1- and Kruppel-like transcription factors. Genome Biol. 4:206.

Kakumani PK, Malhotra P, Mukherjee SK, Bhatnagar RK. 2014. A draft genome assembly of the army worm, Spodoptera frugiperda. Genomics. 104:134-143.

Kalyaanamoorthy S, Minh BQ, Wong TKF, von Haeseler A, Jermiin LS. 2017. Modelfinder: fast model selection for accurate phylogenetic estimates. Nat Methods. 14:587-589.

Kant M, Jonckheere W, Knegt B, Lemos F, Liu J, et al. 2015 Mechanisms and ecological consequences of plant defence 
induction and suppression in herbivore communities. Ann Bot. 115:1015-1051.

Katoh K, Standley DM. 2013. Mafft multiple sequence alignment software version 7: Improvements in performance and usability. Mol Biol Evol. 30:772-780.

Kawahara AY, Plotkin D, Espeland M, Meusemann K, Toussaint EF, et al. 2019. Phylogenomics reveals the evolutionary timing and pattern of butterflies and moths. Proc Natl Acad Sci USA. 116: 22657-22663.

Kennedy MW, Chalamalasetty RB, Thomas S, Garriock RJ, Jailwala P, et al. 2016. Sp5 and Sp8 recruit $\beta$-catenin and Tcf1-Lef1 to select enhancers to activate Wnt target gene transcription. Proc Natl Acad Sci USA. 113:3545-3550.

Kergoat GJ, Goldstein PZ, Le Ru B, Meagher RL, Zilli A, et al. 2021. A novel reference dated phylogeny for the genus Spodoptera guenée (Lepidoptera: Noctuidae: Noctuinae): New insights into the evolution of a pest-rich genus. Mol Phylogenet Evol. 161:107161.

Kim EJ, Kim SJ, Park CJ, Nam YK. 2019. Characterization of testis-specific serine/threonine kinase 1-like (TSSK1-like) gene and expression patterns in diploid and triploid pacific abalone (Haliotis discus hannai; Gastropoda; Mollusca) males. PLoS One. 14: e0226022.

Kosiol C, Goldman N. 2005. Different versions of the dayhoff rate matrix. Mol Biol Evol. 22:193-199.

Koutsos AC, Blass C, Meister S, Schmidt S, MacCallum RM, et al. 2007. Life cycle transcriptome of the malaria mosquito anopheles gambiae and comparison with the fruitfly drosophila melanogaster. Proc Natl Acad Sci USA. 104:11304-11309.

Kriventseva EV, Kuznetsov D, Tegenfeldt F, Manni M, Dias R, et al. 2019. Orthodb v10: sampling the diversity of animal, plant, fungal, protist, bacterial and viral genomes for evolutionary and functional annotations of orthologs. Nucleic Acids Res. 47: D807-D811.

Kück P, Longo GC. 2014. Fasconcat-g: extensive functions for multiple sequence alignment preparations concerning phylogenetic studies. Front Zool. 11:81.

Kück P, Meusemann K, Dambach J, Thormann B, von Reumont BM, et al. 2010. Parametric and non-parametric masking of randomness in sequence alignments can be improved and leads to better resolved trees. Front Zool. 7:10.

Langmead B, Salzberg SL. 2012. Fast gapped-read alignment with bowtie 2. Nat Methods. 9:357-359.

Le Ru B, Barbut J, Capdevielle-Dulac C, Goftishu M, Kergoat GJ. 2018. Re-establishment of Spodoptera teferii laporte in rougeot (lepidoptera: Noctuidae, noctuinae), with an updated molecular phylogeny for the genus Spodoptera guenée. Annal Soc Entomol France (NS). 54:497-510.

Le SQ, Dang CC, Gascuel O. 2012. Modeling protein evolution with several amino acid replacement matrices depending on site rates. Mol Biol Evol. 29:2921-2936.

Le SQ Gascuel O. 2008. An improved general amino acid replacement matrix. Mol Biol Evol. 25:1307-1320.

Li B, Dewey CN. 2011. RSEM: accurate transcript quantification from RNA-seq data with or without a reference genome. BMC Bioinformatics. 12:323.

Li H, Jiang W, Zhang Z, Xing Y, Li F. 2013. Transcriptome analysis and screening for potential target genes for rnai-mediated pest control of the beet armyworm, Spodoptera exigua. PLoS One. 8: e65931.

Li X, Schuler MA, Berenbaum MR. 2007. Molecular mechanisms of metabolic resistance to synthetic and natural xenobiotics. Annu Rev Entomol. 52:231-253.
Liu H, Lan T, Fang DGF, Wang, H Guo, W, et al. 2019. Chromosome level draft genomes of the fall armyworm, Spodoptera frugiperda (Lepidoptera: Noctuidae), an alien invasive pest in China. bioRxiv. 671560; doi: 10.1101/671560

Liu PC, Hao DJ, Hu HY, Wei JR. 2020. Sexual dimorphism and sex-biased gene expression in an egg parasitoid species, Anastatus disparis. BMC Genomics. 21:492.

Loman NJ, Quick J, Simpson JT. 2015. A complete bacterial genome assembled de novo using only nanopore sequencing data. Nat Methods. 12:733-735.

Love MI, Huber W, Anders S. 2014. Moderated estimation of fold change and dispersion for RNA-seq data with DESeq2. Genome Biol. 15:550

Lumley LM, Sperling FAH. 2010. Integrating morphology and mitochondrial DNA for species delimitation within the spruce budworm (Choristoneura fumiferana) cryptic species complex (Lepidoptera: Tortricidae). Syst Entomol. 35:416-428.

Machado V, Serrano J, Galian J. 2016. Identification and characterization of pathogen-response genes (repat) in Spodoptera frugiperda (Lepidoptera: Noctuidae). Folia Biol (Krakow). 64:23-29.

McCulloch KJ, Koenig KM. 2020. Kruppel-like factor/specificity protein evolution in the spiralia and the implications for cephalopod visual system novelties. Proc Biol Sci. 287:20202055.

Mehrkhou F, Talebi AA, Moharramipour S, Naveh VH. 2012. Demographic parameters of Spodoptera exigua (Lepidoptera: Noctuidae) on different soybean cultivars. Environ Entomol. 41: 326-332.

Merkx-Jacques M, Despland E, Bede JC. 2008. Nutrient utilization by caterpillars of the generalist beet armyworm, Spodoptera exigua. Physiol Entomol. 33:51-61.

Misof B, Misof K. 2009. A Monte Carlo approach successfully identifies randomness in multiple sequence alignments: a more objective means of data exclusion. Syst Biol. 58:21-34.

Mitchell A, Mitter C, Regier JC. 2006. Systematics and evolution of the cutworm moths (Lepidoptera: Noctuidae): evidence from two protein-coding nuclear genes. Syst. Entomol. 31:21-46.

Muhlia-Almazán A, Sánchez-Paz A, García-Carreño FL. 2008. Invertebrate trypsins: a review. J Comp Physiol B. 178:655-672.

Müller C, Vogel H, Heckel DG. 2017. Transcriptional responses to short-term and long-term host plant experience and parasite load in an oligophagous beetle. Mol Ecol. 26:6370-6383.

Muller T, Vingron M. 2000. Modeling amino acid replacement. J Comput Biol. 7:761-776

Nam K, Nhim S, Robin S. et al. 2020. Positive selection alone is sufficient for whole genome differentiation at the early stage of speciation process in the fall armyworm. BMC Evol Biol. 20:152. https: //doi.org/10.1186/s12862-020-01715-3.

Nandakumar S, Ma H, Khan AH. 2017. Whole-genome sequence of the spodoptera frugiperda sf9 insect cell line. Genome Announcements. 5:e00829-00817.

Mitchell A, Mitter C, Regier JC. 2006. Systematics and evolution of the cutworm moths (Lepidoptera: Noctuidae): evidence from two protein-coding nuclear genes. Syst. Entomol. 31:21-46.

Navarro-Cerrillo G, Hernández-Martínez P, Vogel H, Ferré J, Herrero S. 2013. A new gene superfamily of pathogen-response (repat) genes in lepidoptera: classification and expression analysis. Comp Biochem Physiol B Biochem Mol Biol. 164:10-17.

Nguyen L-T, Schmidt HA, Von Haeseler A, Minh BQ. 2015. IQ-tree: a fast and effective stochastic algorithm for estimating maximum-likelihood phylogenies. Mol Biol Evol. 32:268-274.

Omkar. 2016. Ecofriendly Pest Management for Food Security. Academic Press. 
Orsucci M, Audiot P, Dorkeld F, Pommier A, Vabre M, et al. 2018. Larval transcriptomic response to host plants in two related phytophagous lepidopteran species: implications for host specialization and species divergence. BMC Genomics. 19:265.

Ou J, Deng HM, Zheng SC, Huang LH, Feng QL, et al. 2014. Transcriptomic analysis of developmental features of Bombyx mori wing disc during metamorphosis. BMC Genomics. 15:820.

Pearce SL, Clarke DF, East PD, Elfekih S, Gordon K, et al. 2017. Genomic innovations, transcriptional plasticity and gene loss underlying the evolution and divergence of two highly polyphagous and invasive helicoverpa pest species. BMC Biol. 15:63.

Pogue M. 2002. World revision of the genus Spodoptera guenée (Lepidoptera: Noctuidae). Mem Am Entomol Soc. 43:1-202.

Price DR, Gatehouse JA. 2008. RNAi-mediated crop protection against insects. Trends Biotechnol. 26:393-400.

Regier JC, Mitter C, Mitter K, Cummings MP, Bazinet AL, et al. 2017. Further progress on the phylogeny of noctuoidea (Insecta: Lepidoptera) using an expanded gene sample. Syst Entomol. 42: 82-93.

Renuka P, Madhav MS, Padmakumari AP, Barbadikar KM, Mangrauthia SK, et al. 2017. RNA-seq of rice yellow stem borer Scirpophaga incertulas reveals molecular insights during four larval developmental stages. G3 (Bethesda). 7:3031-3045.

Robinson GS, Ackery PR, Kitching IJ, Beccaloni GW, Hernández LM. 2010a. Hosts-a database of the world's lepidopteran hostplants. Natural History Museum, London. http://www.nhm.ac.uk/hosts.

Robinson MD, McCarthy DJ, Smyth GK. 2010b. Edger: a bioconductor package for differential expression analysis of digital gene expression data. Bioinformatics. 26:139-140.

Robinson MD, Oshlack A. 2010. A scaling normalization method for differential expression analysis of RNA-seq data. Genome Biol. 11:R25.

Rotllant G, Nguyen TV, Sbragaglia V, Rahi L, Dudley KJ, et al. 2017. Sex and tissue specific gene expression patterns identified following de novo transcriptomic analysis of the Norway lobster, Nephrops norvegicus. BMC Genomics. 18:622.

Sayers EW, Beck J, Brister JR, Bolton EE, Canese K, et al. 2020. Database resources of the national center for biotechnology information. Nucleic Acids Res. 48:D9-D16.

Schulz R. 2004. Field studies on exposure, effects, and risk mitigation of aquatic nonpoint-source insecticide pollution: a review. J Environ Qual. 33:419-448.

Scott JG, Michel K, Bartholomay LC, Siegfried BD, Hunter WB, et al. 2013. Towards the elements of successful insect RNAi. J Insect Physiol. 59:1212-1221.

Seppey M, Manni M, Zdobnov EM. 2019. BUSCO: Assessing Genome Assembly and Annotation Completeness. In: Kollmar M. (eds) Gene Prediction. Methods in Molecular Biology, vol 1962. Humana, New York, NY. 10.1007/978-1-4939-9173-0_14

Smith TF. 2008. Diversity of WD-repeat proteins. In: CS Clemen, L Eichinger, V Rybakin, editors. The Coronin Family of Proteins. New York: Subcellular Biochemistry.
Smits PH, Van De Vrie M, Vlak JM. 1986. Oviposition of beet armyworm (Lepidoptera: Noctuidae) on greenhouse crops. Environ Entomol. 15:1189-1191.

Soubrier J, Steel M, Lee MS, Der Sarkissian C, Guindon S, et al. 2012. The influence of rate heterogeneity among sites on the time dependence of molecular rates. Mol Biol Evol. 29: 3345-3358.

Tian L, Zeng Y, Xie W, Wu Q, Wang S, et al. 2019. Genome-wide identification and analysis of genes associated with RNA interference in Bemisia tabaci. Pest Manag Sci. 75:3005-3014.

Truman JW, Riddiford LM. 2019. The evolution of insect metamorphosis: a developmental and endocrine view. Philos Trans R Soc Lond B Biol Sci. 374:20190070.

Veerassamy S, Smith A, Tillier ER. 2003. A transition probability model for amino acid substitutions from blocks. J Comput Biol. 10:997-1010.

Volney WJA, Fleming RA. 2007. Spruce budworm (Choristoneura spp.) biotype reactions to forest and climate characteristics. Global Change Biol. 13:1630-1643.

Walker BJ, Abeel T, Shea T, Priest M, Abouelliel A, et al. 2014. Pilon: an integrated tool for comprehensive microbial variant detection and genome assembly improvement. PLoS One. 9:e112963.

Wheeler W. 2002. Pesticides in Agriculture and the Environment. New York: CRC Press. 10.1201/9780203909430

Whelan S, Goldman N. 2001. A general empirical model of protein evolution derived from multiple protein families using a maximum-likelihood approach. Mol Biol Evol. 18:691-699.

Wickham H. 2016. Ggplot2: Elegant Graphics for Data Analysis. New York: Springer

Xiao H, Ye X, Xu H, Mei Y, Yang Y, et al.. 2020. The genetic adaptations of fall armyworm Spodoptera frugiperda facilitated its rapid global dispersal and invasion. Mol Ecol Resour. 20:1050-1068. 10.1111/1755-0998.13182 32359007

Xue X-Y, Mao Y-B, Tao X-Y, Huang Y-P, Chen X-Y. 2012. New approaches to agricultural insect pest control based on RNA interference. In: Advances in Insect Physiology. Elsevier. p. 73-117.

Yang Z. 1994. Maximum likelihood phylogenetic estimation from DNA sequences with variable rates over sites: Approximate methods. J Mol Evol. 39:306-314.

Young MD, Wakefield MJ, Smyth GK, Oshlack A. 2010. Gene ontology analysis for RNA-seq: accounting for selection bias. Genome Biol. 11:R14.

Zhang F, Zhang J, Yang Y, Wu Y. 2020. A chromosome-level genome assembly for the beet armyworm (Spodoptera exigua) using PacBio and Hi-C sequencing. bioRxiv.2019. 10.1101/2019.12.26.889121

Zhou CY, Zha XF, Liu C, Han MJ, Zhang LY, et al. 2016. Identification of mbf2 family genes in Bombyx mori and their expression in different tissues and stages and in response to Bacillus bombysepticus infection and starvation. Insect Sci. 23:502-512.

Communicating editor A. Seetharam 